\title{
Evidence of an association between neurological manifestations (microcephaly and GBS) and Zika virus infection: the usefulness of ecological studies with syndromic surveillance data
}

\author{
Patrícia Brasil $^{1}$, Mayumi D. Wakimoto ${ }^{1}$, Carlos Brito ${ }^{2}$ \\ ${ }^{1}$ Fundação Oswaldo Cruz, Rio de Janeiro, RJ, Brazil; ${ }^{2}$ Universidade Federal de Pernambuco, Recife, PE, Brazil \\ Correspondence to: Patrícia Brasil. Fundação Oswaldo Cruz, Rio de Janeiro, RJ, Brazil. Email: patricia.brasil@ini.fiocruz.br. \\ Provenance: This is a Guest Editorial commissioned by the Editor-in-Chief Baoli Zhu (Jiangsu Provincial Center for Disease Control and Prevention, \\ Nanjing, China). \\ Comment on: Paploski IA, Prates AP, Cardoso CW, et al. Time Lags between Exanthematous Illness Attributed to Zika Virus, Guillain-Barré \\ Syndrome, and Microcephaly, Salvador, Brazil. Emerg Infect Dis 2016;22:1438-44.
}

Received: 05 June 2017; Accepted: 06 June 2017; Published: 03 July 2017.

doi: 10.21037/jphe.2017.06.03

View this article at: http://dx.doi.org/10.21037/jphe.2017.06.03

\section{Introduction}

The first relevant clinical and epidemiological Zika outbreaks were described in 2007 in the Yap Islands (1) and six years later in French Polynesia, with an estimate of 29,000 cases occurring in the period from October 2013 to February 2014 (2). In late 2014, cases of acute exanthematous illness (AEI), involving widespread rash of unclear etiology, were reported in several municipalities in northeastern Brazil. The identification of Zika virus (ZIKV) occurred in May of the same year and subsequent expansion to other regions was observed. ZIKV infection has been reported in Rio de Janeiro since January 2015 (3).

It was only after the Brazil outbreak in 2015 that awareness of ZIKV circulation and the potential for severe disease outcomes was revealed. The disease quickly spread to other countries and within one year 49 countries in the Americas reported autochthonous cases. From 2015 to May 2017, 563,168 Zika autochthonous cases were reported in the Americas, of which 40\% (223,230 cases) occurred in Brazil (4). Advanced diagnostic techniques and surveillance systems allowed the investigation and reporting of autochthonous cases.

Neurological complications related to Zika were first described after the outbreak of French Polynesia in 2013. There are currently 22 countries with reports of cases potentially associated with infection, although the total number of cases is uncertain (5). In 2015, the analysis of
1,474 suspected cases of Guillain-Barré syndrome (GBS) associated with Zika reported in seven Latin American countries revealed a 2 - to 8 -fold increase in incidence compared to that reported in years prior to the epidemic (6).

Since October 2015, when the hypothesis of a ZIKV association with microcephaly was raised in northeastern Brazil, 3,130 cases of congenital infection associated with the virus have been reported in Latin America until the last bulletin of PAHO in 2017. Of these, 2,653 (85\%) were reported in Brazil, but only 863 (32.5) were confirmed by laboratory criteria. Brazil uses a different classification system and considers confirmed cases those defined as probable or laboratory confirmed cases (4).

\section{Official data and non-official data}

Although ZIKV was isolated in April 2015, it was not until February 2016 that the official surveillance system of the Ministry of Health of Brazil referred to Zika as a reportable disease (7). Thus, there is a large gap in Zika cases estimates in the first major epidemic in the Americas, which is important for the analysis of the disease pattern as well as for estimating the risk of complications associated with infection such as GBS and congenital syndrome.

Despite the simultaneous circulation of more than one arbovirus (8-10), epidemics tend to produce different patterns of incidence each year, with the predominance of one arbovirus over the others. Cardoso analyzed 14,835 
cases of undetermined exanthematous disease in Salvador, and despite finding only 3 positive samples for Zika by RTPCR, he considered the epidemic as virus-related due to the low frequency of symptoms such as fever and arthralgia common in dengue and chikungunya cases (9).

In Pernambuco, also in northeastern Brazil and another epicenter of the epidemic as well as of neurological complications, the official registries of the surveillance system reported dengue fever as the arboviral disease responsible for most cases of the epidemic $(>140,000)$ and $<2,000$ suspected Zika cases. However, a hospital-based study in the state capital analyzed 1,046 records of suspected cases of arboviruses, classifying $84 \%$ of them as probable cases of Zika and only $14 \%$ as dengue, based on clinical criteria (11).

The low sensitivity of the Zika case definitions in Brazil results in many cases being erroneously attributed to dengue fever. When using clinical criteria for the definition of a suspected case of dengue, $48 \%$ of confirmed cases of Zika ( $\mathrm{n}=138)$ in a cohort of Rio de Janeiro would have been reported as dengue to the surveillance system (Brasil $\mathrm{P}$-unpublished data). The misreporting of arboviral cases has serious consequences not only for surveillance but also for patient management and understanding of the pathogenesis of disease.

\section{The importance of syndromic surveillance for detecting and monitoring epidemics of emerging diseases}

Syndromic surveillance plays a significant role in increasing the sensitivity for reporting suspected cases based on signs and symptoms, especially considering the low availability of laboratory tests for diagnostic confirmation in routine health services.

In the study of Paplosky et al. published in EID in 2016, the authors utilized data from the surveillance system of the Centers for Information and Epidemiologic Surveillance of Salvador (CIES) established in public emergency health centers as sentinel units for systematic surveillance of patients with AEI of unknown cause in Salvador. They used the presence of rash, with or without fever, from patients whose clinical and epidemiological characteristics did not meet the clinical criteria compatible with dengue, chikungunya, measles or rubella, to define ZIKV infection. To assess neurological syndromes potentially associated with ZIKV they used surveillance of hospitalizations due to neurological manifestations such as GBS and variants (e.g., Miller-Fisher syndrome). Regarding microcephaly, all suspected cases (criterion to an occipitofrontal perimeter $<32 \mathrm{~cm}$ ) were analyzed, which totaled less than $30 \%$ of the ZIKV cases. Epidemiological curves were based on 3 and 5 week moving averages. The main focus was to estimate the intervals with significant correlation between reported cases of AEI and cases of GBS and microcephaly, covering the gestational period (0-40 weeks).

\section{A need for an accurate case-definition}

Oversensitive and non-specific case definitions can lead to an overestimate of ZIKV infectious burden while attributing to it a lower amount of complications such as GBS or congenital ZIKV syndrome.

A significant number of cases of the Paploski's study may not meet the criteria of suspected cases advocated by PAHO and the Ministry of Health of Brazil, necessary for notification by the surveillance system, as has occurred in other studies $(3,9,11)$, in which fever and arthralgia were present in less than $40 \%$ of laboratory-confirmed ZIKV infection.

As ZIKV laboratory diagnosis still relies on the use of expensive and complex molecular techniques with low sensitivity, due to a narrow window of detection, most suspected cases are not confirmed by these tests, mainly reserved for pregnant women.

An accurate definition of a suspected ZIKV case would permit the prioritization of high-risk groups such as pregnant women, and the monitoring of complications. Furthermore, it would be crucial to the surveillance system to gauge the magnitude of an epidemic and also useful for surveillance of imported cases in non-endemic areas.

\section{Contribution of ecological studies to the evidence of association between ZIKV infection and microcephaly and GBS}

Based on the clinical manifestations potentially associated with Zika, studies in the northeast of Brazil, the epicenter of the epidemic, have sought to analyze the epidemic of 2015 from secondary data.

Using raw and smoothed temporal data collected during the outbreak Paplosky et al. demonstrated a clear temporal correlation between the exanthematous disease that occurred in early 2015 and the cases of GBS and microcephaly, strongly suggesting that the epidemic was due to Zika. Their findings confirm preliminary results from the study by Oliveira et al., in which the authors found a 23-week interval between GBS and microcephaly peaks in 
2015, and found that the time course of GBS cases (based on hospital reports) was probably more accurate than that of the reportable registers system, which was irregularly used in 2015. Both studies $(12,13)$ suggested that the AEI outbreak preceded a 5-9 week interval of the GBS cases, which coincides with the peak of the disease, occurred in the first four months of 2015. Their results complement the case-control study which reported an association of GBS with ZIKV in French Polynesia (14).

The number of suspected cases of microcephaly peaked after a lag of 30-33 weeks from the peak in AEI cases, which corresponded to potential infections of mothers during the first trimester of gestation (7-8 months before giving birth). According to the authors, these findings demonstrated a strong temporal association between potential exanthematous disease in the first trimester of pregnancy and birth outcome, although adverse outcomes after ZIKV infection may occur regardless of the timing of maternal infection as has been shown in a longitudinal cohort study in Rio de Janeiro (15).

\section{Conclusion remarks}

The emergence of a new disease poses numerous challenges ranging from an accurate clinical diagnosis, laboratory methods for diagnosis confirmation, to the modes of transmission, and control. The syndromic surveillance strategy allows the collection of information with sufficient sensitivity to monitor a disease that is not included in the list of compulsory reportable diseases. The systematic surveillance of patients with acute exanthematous disease of unknown origin allowed the recognition of the ZIKV epidemic in Salvador, Recife, and Rio de Janeiro. The association between ZIKV infection and microcephaly epidemics first raised by Brito in 2015 in Recife was confirmed by Paplosky et al. by temporal series, which demonstrated time lags between the epidemiologic curves of the suspected ZIKV infection outbreak, reported cases of GBS, and reported suspected cases of microcephaly in Salvador.

\section{Acknowledgements}

None.

\section{Footnote}

Conflicts of Interest: The authors have no conflicts of interest to declare.

\section{References}

1. Duffy MR, Chen TH, Hancock WT, et al. Zika virus outbreak on yap island, federated states of Micronesia. $\mathrm{N}$ Engl J Med 2009;360:2536-43.

2. European Center for Disease Prevention and Control (ECDC). Rapid risk assessment: Zika virus infection outbreak, French Polynesia. Stockholm: ECDC; 2014:12.

3. Brasil P, Calvet GA, Siqueira AM et al. Zika virus outbreak in Rio de Janeiro, Brazil: clinical characterization, epidemiological and virological aspects. PLoS Negl Trop Dis 2016;10:e0004636.

4. PAHO WHO a. Zika cumulative cases. [cited 2017 May 25]. Available online: http://www.paho.org/hq/index. php?option=com_content $\&$ view $=$ article $\&$ id $=12390 \&$ Itemi $\mathrm{d}=42090$ \&lang $=$ en

5. PAHO WHO b. Regional Zika Epidemiological Update (Americas). [cited 2017 April 27]. Available online: http:// www.paho.org/hq/index.php?option=com_content\&view= article $\&$ id $=11599 \&$ Itemid $=41691$ \&lang $=$ en

6. Dos Santos T, Rodriguez A, Almiron M, et al. Zika virus and the Guillain-Barré syndrome - case series from seven countries. N Engl J Med 2016;375:1598-601.

7. Brazil 2016. Ministério da saúde. Secretaria de Vigilância em Saúde. Procedimentos a serem adotados para a vigilância da Febre do vírus Zika no Brasil. 2016:7. [cited 2017 April 27]. Available online: http://portalsaude.saude. gov.br/images/pdf/2016/marco/07/Nota-Informativazika.pdf

8. Monitoramento dos casos de dengue, febre de chikungunya e febre pelo vírus Zika até a Semana Epidemiológica 15, 2017. [cited 2017 April 27]. Available online: http:// portalarquivos.saude.gov.br/images/pdf/2017/maio/05/ Monitoramento-dos-casos-de-dengue-febre-dechikungunya-e-febre-pelo-virus-Zika-ate-a-SemanaEpidemiologica.pdf

9. Cardoso CW, Paploski IA, Kikuti M, et al. Outbreak of exanthematous illness associated with Zika, chikungunya, and dengue viruses, salvador, Brazil. Emerg Infect Dis 2015;21:2274-6.

10. Pessôa R, Patriota JV, Lourdes de Souza Md, et al. Investigation Into an Outbreak of Dengue-like Illness in Pernambuco, Brazil, Revealed a Cocirculation of Zika, Chikungunya, and Dengue Virus Type 1. Medicine (Baltimore) 2016;95:e3201.

11. Brito CA, Brito CC, Oliveira AC, et al. Zika in 
pernambuco: rewriting the first outbreak. Rev Soc Bras Med Trop 2016;49:553-8.

12. De Oliveira WK, Espinal MA, Dye C. Zika virus infection and associated neurologic disorders in Brazil. N Engl J Med 2017;376:1591-3.

13. Paploski IA, Prates AP, Cardoso CW, et al. Time Lags between Exanthematous Illness Attributed to Zika Virus, Guillain-Barre Syndrome, and Microcephaly, Salvador,

doi: 10.21037/jphe.2017.06.03

Cite this article as: Brasil $\mathrm{P}$, Wakimoto MD, Brito C. Evidence of an association between neurological manifestations (microcephaly and GBS) and Zika virus infection: the usefulness of ecological studies with syndromic surveillance data. J Public Health Emerg 2017;1:64.
Brazil. Emerg Infect Dis 2016;22:1438-44.

14. Besnard M, Lastère $S$, Teissier A, et al. Evidence of perinatal transmission of Zika virus, French Polynesia, December 2013 and February 2014. Euro Surveill 2014;19. pii: 20751.

15. Brasil P, Pereira JP, Moreira ME, et al. Zika virus infection in pregnant women in Rio de janeiro. $\mathrm{N}$ Engl J Med 2016;375:2321-34. 